\title{
CLASSIFICAÇÃO DE GESTOS USANDO ELETROMIOGRAFIA PARA CONTROLE DE DISPOSITIVOS
}

\author{
Arthur M. Martins ${ }^{1}$, Fernanda S. Andrade ${ }^{1}$, Gabriel S. Nascimento ${ }^{1}$, Gustavo B. \\ Coutinho' ${ }^{1}$ Moises F. Queiroz ${ }^{1}$, Ricardo A. Valentim ${ }^{2}$, Danilo P. Nagem ${ }^{2}{ }^{1}$ Universidade \\ Federal do Rio Grande do Norte (UFRN) - Natal, RN - Brazil \\ ${ }^{2}$ Universidade Federal do Rio Grande do Norte (UFRN) - Natal, RN - Brazil \\ abmm arthurehotmail.com, fernanda sena@ufrn.edu.br, \\ gabriel_iañ207@hotmail.com, gustavo.coutinho@lais.huol.ufrn.br, \\ moisesfreitas00174@gmail.com, ricardo.valentim@ufrnet.br, \\ nagemeufrnet.br
}

\begin{abstract}
This study provides an electromyography (EMG) analysis to recognize non conventional positions of the Myo ${ }^{T M}$ Armband and expand the gesture pattern list in order to control devices more accurately and with more functionality. Among the controls, a library of commands has been developed that can be applied in several different projects, such as man-machine interface for games, control of multimedia devices, loT projects, control of home appliances or robotic prostheses. The system makes it possible to control any device through muscular contraction.
\end{abstract}

Resumo. Este estudo aborda a análise da eletromiografia (EMG) para reconhecer posições não convencionais do $\mathrm{Myo}^{\mathrm{TM}}$ Armband e expandir a lista de padrões de gestos, a fim de controlar dispositivos de forma mais precisa e com mais funcionalidades. Dentre os controles, foi desenvolvido uma biblioteca de comandos que podem ser aplicados em diversos projetos diferentes, como a Interface Homem-Máquina para jogos, controle de dispositivos de multimídia, projetos IoT, controle de eletrodomésticos ou de próteses robóticas. $O$ sistema possibilita o controle de qualquer dispositivo através da contração muscular.

\section{Introdução}

Os sinais mioelétricos (SME), são sinais biológicos que podem ser medidos por eletrodos sobre os músculos, são sensores que identificam a despolarização da membrana muscular e induzem a sua contração (DE LUCA, 1979), ou seja, é consequência de um controle cerebral sobre os músculos. Muitos desses eletrodos podem ser utilizados sobre a pele do indivíduo. Os eletrodos de superfície capturam sinais de vários músculos de uma vez e são menos precisos que os implantados, porém apresentam um menor custo e não são invasivos. Outro problema com os eletrodos de superfície é o cross talking, isto é, a ativação de um músculo pode interferir no sinal de outro músculo não sendo possível mensurar com exatidão a contração correta e sua intensidade. Para isso o uso de diversos eletrodos sobre a superfície permite uma melhor análise dos sinais. Com o uso de diversos sensores mioelétricos ao mesmo tempo sobre a superfície é possível correlacionar esses sinais não só a um músculo, mas a todo um movimento físico, como um abrir de mão. Um dos dispositivos para medição de sinais mioelétricos presentes no mercado é o Myo ${ }^{\mathrm{TM}}$ Armband, desenvolvido pela Thalmic Labs, que é capaz de identificar oito canais de captação de 
sinais mioelétricos (EMG), sendo ainda munido de um giroscópio, um acelerômetro e de comunicação sem fio. Com o uso desse bracelete é possível ao usuário o controle de dispositivos a distância utilizando apenas a contração muscular e movimento de seus membros superiores. A biblioteca de comandos pode ser aplicada em diversos projetos diferentes, como a interface Homem-Máquina para jogos, controle de dispositivos de multimídia, projetos loT, controle de eletrodomésticos ou de próteses robóticas. O sistema possibilita que um indivíduo com amputação no membro ainda consiga relizar essas atividades, pois ainda há contração muscular na área do coto. Assim ele irá conseguir realizar atividades comuns do dia a dia, como segurar objetos, cumprimentar pessoas ou ainda utilizar os projetos de tecnologia sugeridos, e assim, possuindo também, um papel fundamental na inclusão e independência do indivíduo na sociedade.

\section{Descrição do problema}

Uma pessoa que possui uma amputação de membro superior necessita de um trabalho de acessibilidade e de assistência para acontecer o processo de inclusão do indivíduo na sociedade. Para isto, existem diversos projetos e dispositivos de mercado com esta finalidade de tecnologia assistiva. O Myo ${ }^{\mathrm{TM}}$ Armband é um dispositivo que permite ao usuário controlar aplicações sem fio usando vários movimentos da mão, porém ele possui uma API limitada a apenas 6 gestos diferentes. Dependendo do projeto será necessário um maior número de gestos, para um controle maior de funções.

Assim, foi considerado a oportunidade de expandir esses gestos do $\mathrm{Myo}^{\mathrm{TM}}$ Armband, para expandirmos também o número de projetos de tecnologia assistiva voltados para pacientes amputados. Além de posteriormente tornar possível a construção de um sensor mioelétrico com mais precisão e com a finalidade de representar de fato todo o movimento real humano, ou seja, que interprete toda a anatomia muscular humana com exatidão.

Para o desenvolvimento de um sistema próprio de sensores mioelétricos mais preciso, e que desempenhe o objetivo de detectar exatamente movimentos mais minuciosos do corpo humano, como por exemplo: A detecção da extensão e flexão de apenas um dedo, sem identificar a EMG responsável por outro movimento, é necessário um estudo da anatomia humana e como seria um conjunto de sensores e um tratamento de dados inteligentes o suficiente para separar cada movimento especifico de cada dedo.

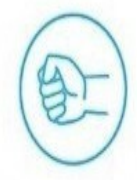

Make Fist

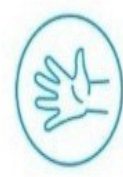

Spread Fingers

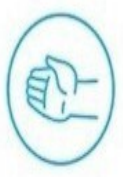

Wave Out

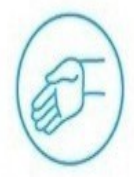

Wave In

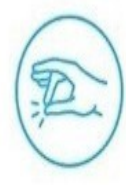

Double-Tap

Figura 1. Gestos padrões da API do Myo ${ }^{\mathrm{TM}}$ Armband

\section{Relevância}

O sistema pode abrir oportunidades para outros projetos de baixo custo na área da saúde, afim de revolucionar e substituir os produtos caros obtidos pelo governo. Para teste do sistema foi desenvolvido uma prótese robótica de baixo custo, 
e ao ser controlada pelo sensor, ela pôde desempenhar todas as funções necessárias para um paciente com amputação. O objetivo é aprimorar o projeto para que ela seja distribuida no Sistema Único de Saúde, pois próteses robóticas como esta custam caro em Universidades especializadas nos Estados Unidos.

\section{Mercado/Público alvo}

No Laborátorio de Inovação Tecnológica em Saúde (LAIS) localizado no Hospital Universitário Onofre Lopes (HUOL - UFRN) é comum ver alguns pacientes com amputação, e o objetivo do projeto é atender justamente essas pessoas que não possuem condições financeiras de adquirir uma prótese robótica, que se movimente e as ajude no dia a dia; ou que não enxergam a possibilidade de ter acesso a uma tecnologia de baixo custo, como o controle de um eletrodoméstico na propria casa, à distancia.

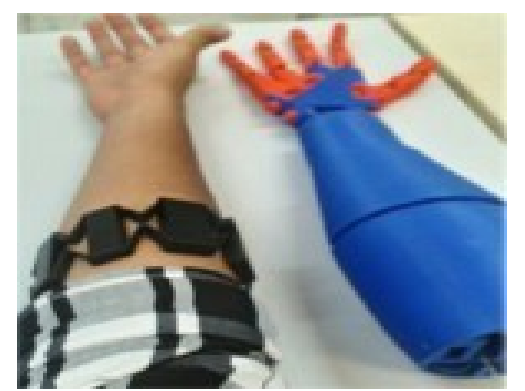

Figura 2. Prótese robótica

\section{Soluções existentes}

Em uma comunicação Bluetooth entre o $\mathrm{Myo}^{\mathrm{TM}}$ Armband e o controlador (Raspberry $\mathrm{PI}$ ), foi desenvolvida uma interface de calibração que funciona como um aprendizado de máquina, onde as variações dos sinais são quantizadas e armazenadas em arquivos DAT (.dat), esses arquivos são identificados de acordo com a posição em que foi calibrada. Quanto mais tempo calibrando uma posição, mais ela se torna precisa, e assim, foi possível obter uma rotina e identificar até 10 tipos diferentes de ações motoras.

Para aplicação do sistema foi utilizado a tecnologia de impressão 3D para a produção de uma prótese robótica feita a partir de uma adaptação do projeto Open Source de impressão 3D InMoov. A movimentação da prótese se mostrou muito eficiente, porém estudos mais precisos e com técnicas de avaliações mais apuradas na análise dos sinais já estão em andamento no LAIS. Assim como um desenvolvimento de possíveis projetos com essa aplicação, um software de calibração mais preciso e otimizado também está sendo desenvolvido. É esperado que em um futuro haja um aumento do número de movimentos possíveis, e assim, que nós possamos reproduzir todos os gestos reais e com as limitações apenas de um humano.

Outra solução ideal seria abandonar a dependência do Myo ${ }^{\mathrm{TM}}$ Armband, pois ele é um produto de mercado, e utilizar sensores mioelétrico comuns de eletrônica, como o AT-04-001. Porém, seria necessário um estudo de arquitetura do sistema, pois é sabido que esse tipo de sensor comum possui uma precisão bem baixa em relação ao da primeira solução. 


\section{Oportunidades para avaliação futura de uma proposta de solução}

A idéia é ampliar o número de aplicações de Tecnologia Assistiva usando sensores EMG, para tornar a área que se preocupa com pessoas com membros amputados mais visível. E para isto, é preciso melhorar o sistema de comandos, pois os sensores utilizados atualmente ainda são bem imprecisos.

O LAIS é um laboratório situado dentro de um hospital universitário, onde diariamente profissionais e pesquisadores trabalham para realizar desenvolvimento da área da saúde no Brasil por meio da inovação. Nas bases de pesquisa ja encontramos infraestrutura suficiente para desenvolver as aplicações, com espaço e equipamento, que envolvem computadores, componentes eletrônicos, ferramentas e impressoras 3D. É esperado a partir de agora, que aprimoramentos sejam feitos e que de fato seja feito um produto, para o cenário de pesquisas em saúde do Brasil.

\section{Referências}

Akhmadeev K., Rampone E., Yu T., Aoustin Y., Carpentier E. L. A realtime gesture classification using surface EMG to control a robotics hand. ENOC 2017, Jun 2017, Budapest, Hungary. 2017.

De Luca, C. J. (1979). Physiology and mathematics of myoelectric signals. IEEE Transactions on Biomedical Engineering, New York, v. BME-26, n.6, p.313-325.

K. Xing, P. Yang, J. Huang, Y. Wang, Q. Zhu, A real-time EMG pattern recognition method for virtual myoelectric hand control. Neurocomputing, vol. 136, pp. 345355, Jul. 2014.

InMoov Open Source prosthetic hand. Disponível em: https://inmoov.fr/. Acesso em: 2018.

T. L. Inc. Myo connect start page. Disponível em: https://www.myo.com/start. Acesso em: 2017.

Tomaszewski, M. Myo sdk matlab mex wrapper. Disponível em: https://github.com/ marktoma/MyoMex. Acesso em: 2017. 